\title{
Tourisme culturel et Patrimoine Alexandrin Stratégie de développement des Relations Publiques aux Musées Riham El Wakil
}

Instructor, High Institute of Tourism, Hospitality and Computer, Siouf, Alexandria

ARTICLE INFO

\section{Mots clés:}

Alexandrie

Patrimoine

tourisme culturel

musée

relations publiques

stratégie

(JTHH)

Vol. 1 No. 7, (2020)

pp 98-116.

\begin{abstract}
Résumé
Fière de son héritage vivant et implacable par les civilisations qui se sont succédées, Alexandrie reflète sa mémoire patrimoniale à travers ses riches collections renfermées aux musées de la ville. Les musées comme acteurs de leur temps, doivent relever le défi d'être non seulement l'institution qui met en valeur le patrimoine, mais aussi un instrument utile pour accéder à un développement humain équilibré, à un meilleur bien-être collectif dans lequel s'inscrit forcément le tourisme culturel. Le tourisme apparaît ainsi comme un facteur susceptible d'engendrer d'importants bénéfices économiques qui contribuent à gérer et à préserver le patrimoine alexandrin, comme un moyen valable de favoriser le développement global de la communauté locale qui détient ce patrimoine et pour le musée lui-même.

Mettre au jour une stratégie de communication se considère comme une nouvelle restructuration des activités muséales. Dans cette étude le Service des Relations Publiques du musée envisagé comme un système adéquat au profil des publics par lequel la présentation des collections sera en fonction des motivations des visiteurs. Un acte créatif qui envisage le musée comme un organisme vivant et évolutif, qui possède une identité propre, qui vit en interaction avec son milieu, par et pour ses publics, et qui a quelque chose d'essentiel et d'unique à apporter qu'il faut savoir expliciter.
\end{abstract}

\section{Introduction}

Au sein de cet ensemble mondial d'une grande complexité, le tourisme joue un rôle économique essentiel pour certains pays comme l'Égypte, de même que la culture comme moyen idéal de transformer ces flux touristiques en énergie positive. La culture et le patrimoine constituent un élément essentiel de l'offre en matière de tourisme culturel. Les musées de la ville sont les lieux de regards pour le visiteur culturel d'Alexandrie, des lieux de représentation de l'identité de la ville et de constitution du dialogue culturel mutuel où les idées s'échangent et les richesses patrimoniales matérielles de la ville se présentent. Actuellement, l'évolution naturelle de l'activité muséologique demande l'application à la conservation des biens culturels, à l'architecture et à la muséographie. En plus la diversification, la spécialisation et la planification en tant que méthode essentielle et l'émergence de tant d'activités nouvelles. Le développement d'un Service de Relations Publiques responsable, spécialiste pour une meilleure médiation culturelle; une commercialisation efficace des produits et des événements et une élaboration des quêtes clientèles devient une nécessité indispensable au sein des musées alexandrins. 


\section{Importance et objectifs de l'étude}

Les musées jouent un rôle important dans le phénomène touristique à Alexandrie et c'est pourquoi on s'y intéresse de la présente étude pour réfléchir sur la manière de développement de l'activité touristique des musées alexandrins pour avoir un offre culturel de qualité, à proposer un produit qualitatif en terme de tourisme culturel. Il s'agit d'impulser un véritable processus d'appropriation des musées par les publics, d'élaborer une bonne politique qui permettrait d'aboutir à une meilleure relation entre les musées et leurs publics. D'augmenter la fréquentation muséale et rendre les musées alexandrins accessibles à tous publics et d'établir des politiques d'enquêtes auprès des visiteurs et la création ou la modernisation d'un service spécialiste de relations publiques.

\section{Problématique de l'étude}

Les musées d'Alexandrie affrontent aujourd'hui des problèmes qui l'empêchent de remplir les conditions lui permettant d'atteindre ses objectifs en termes de fréquentation publique. Ces handicaps se nomment entre autres : insuffisance des financements, donc des frais d'entretiens des collections, inexistence de recrutement de personnel qualifié, manque cruel d'infrastructures et de structures d'accueil des publics ...etc. Le tourisme culturel de nos jours comporte des exigences et demande beaucoup d'efforts, politiques, économiques et surtout en termes de prestations et services. On aborde par cette étude le problème du service des relations publiques aux musées alexandrins afin de mieux répondre aux exigences du tourisme culturel intérieur et extérieur.

\section{Méthodologie de la recherche}

Les méthodes sont les règles générales qui nous aident à prouver les réalités et les découvertes scientifiques (الثيبانى، Pour mener ce travail, la méthode inductive était utilisée pour prélever les données autour des notions du musée, tourisme culturel et patrimoine. On s'est référé à l'étude documentaire comme outil scientifique de base pour mettre au jour les musées existants à Alexandrie et leurs spécifiés. On a adopté par la suite une démarche analytique des données collectées par l'approche appliquée, par une quête sur un échantillon représentatif des visiteurs afin de prélever des informations qualitatives et quantitatives. La recherche est alors partie du côté théorique représentant les musées de la ville et la problématique de service de relations publiques ; la compréhension des concepts et des liens existants entre eux au côté pratique qui donne du poids et de la crédibilité à l'étude afin de proposer des suggestions viables.

\section{Plan de la recherche}

Pour répondre à la question traitée de la problématique du thème, l'étude marque les corrélations existantes entre tourisme culturel, patrimoine et musée en ces temps de mondialisation et de développement touristique durable. Elle est répartie en trois parties essentielles qui porteront respectivement sur : l'importance d'Alexandrie en terme de potentialités touristiques sur la base de son riche patrimoine; le rôle et le poids des musées de la ville en tant que carrefour d'histoire et de culture et la détermination de la situation actuelle du service des relations publiques aux musées par une quête auprès des visiteurs pour voir dans une sorte d'analyse critique ce qui constitue à l'heure d'aujourd'hui leurs forces et faiblesses qui influent indubitablement sur la réussite ou non de la mission de satisfaction des publics et donc de pôle touristique culturel. Ce qui permettra enfin de suggérer une stratégie muséale de 
relations publiques à Alexandrie afin de déceler les insuffisances qu'il va falloir nécessairement corriger et adapter aux nouvelles exigences muséales .

\section{Limites de la recherche}

Un des facteurs de succès d'une recherche est la définition de ses limites objectives, temporelles

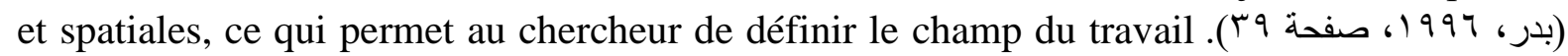
L'étude est effectuée à Alexandrie, deuxième capitale et premier port d'Égypte et l'une des plus importantes stations balnéaires du Moyen-Orient. Connue par la sirène de la Méditerranée, elle s'étend de $70 \mathrm{~km} 2$ sur le littoral Nord-Ouest du Delta du Nil. Bordée au Nord par la Mer Méditerranée et au sud par le lac Mariout, à l'Est par le Golf d'Aboukir et à l'ouest par Sidi Kreir.(1) L'étude portait sur une vue d'ensemble des musées de la ville d'Alexandrie, tel que : le Musée National, le Musée des Bijoux Royaux, les musées de la Bibliothèque d'Alexandrie, le musée des Beaux-Arts, le complexe muséale de Mahmoud Saïd, le musée des études marine et la maison de Cavafy en raison de leur rôle patrimonial important au tourisme culturel intérieur et extérieur. Cependant, le côté pratique de l'étude portait sur la sélection d'un échantillon de visiteurs des musées.

\section{Alexandrie, berceau d'un patrimoine national et international}

Grâce à l'emplacement idéal de la ville à proximité de l'île de Pharos, Alexandrie prend son nom en l'honneur de son fondateur, choisi par les dieux fut fondée par Alexandre Le Grand en 332 avant J.C (Claude, 2001, p. 6). Elle abritait les autochtones, la garde macédonienne, quelques immigrés grecs, ainsi qu'une minorité juive (Errera \& Mourrain , 2001).Favorisée par sa situation géographique et par la construction astucieuse de son port, Alexandrie devint un centre économique et une ville maritime des plus renommées de l'Antiquité. Sous ses successeurs, les Ptolémées, elle devient capitale, le plus grand port du monde antique et un véritable creuset culturel où savants, artistes et philosophes se réunissent dans l'académie des

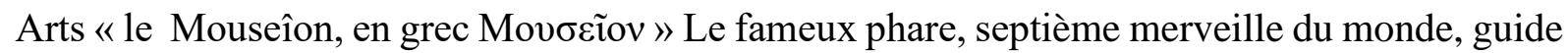
alors les marins avec son signal lumineux visible à plus de $50 \mathrm{~km}$ (Empereur, Alexandrina1, 1998, pp 21-32., 1998).

Elle restait le centre mondial de la culture hellénistique pour trois siècles et prend fin avec la chute de Cléopâtre VII et de son allié Marc-Antoine dans la célèbre bataille d'Actium en l'an 31 Av. J.C (El Wakil, Vers une éducation patrimoniale au service d'un tourisme durable à Alexandrie, 2017, p. 220)Avec le triomphe de l'empereur Octave Auguste, l'Égypte restait un centre de culture grâce à la présence d'universités et d'organismes scientifiques divers et un centre financier important. Alexandrie demeure la capitale de l'Égypte jusqu'à l'arrivée des Arabes en 640 après J.C (Le Gouvernerat d'Alexandrie, 1989). Au début de notre ère, Alexandrie était une cité cosmopolite aux multiples visages peuples, langues et cultures du monde antique s'y rencontrent. Formidable creuset culturel, elle mêle tout à la fois les héritages de la pensée grecque, de la religion pharaonique, le judaïsme, le christianisme et l'islam (Empereur, Alexandrie: hier et demain, 2001, p. 33).Alexandrie, cité bourdonnante d'activité, riche, marchande et industrielle, à la fois égyptienne et méditerranéenne, continue aujourd'hui encore d'essayer de se trouver une identité. Ville littéraire et ville de souvenir, elle possède

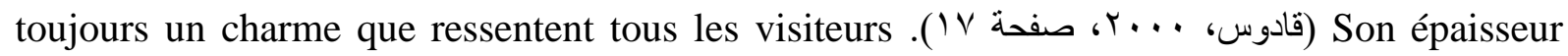
historique s'inscrit encore dans son magnifique paysage, avec sa beauté naturelle transformée par les hommes au cours des millénaires, avec son littoral qui cache encore de somptueuses villas néo-classiques, des catacombes romaines, des vestiges archéologiques, ... etc. Toutes ces 
richesses façonnent les visiteurs culturels à découvrir la magnificence d'Alexandrie dans une quête continue de sa personnalité.

\section{Tourisme culturel, patrimoine et musées}

Le tourisme est précisément l'un de ces mouvements mondiaux qui ont ébranlé les fondements du musée. Un phénomène socioculturel aux influences considérables, aux dimensions multiples; si l'économie est l'une d'entre elle, il en est une autre dont on parle beaucoup à l'heure actuelle, et qui nous concerne directement: sa dimension culturelle (Direction des musées de France, 1994). Le tourisme est une activité culturelle qui est en un rapport direct avec les musées, et le patrimoine. Il est évident que cette forme culturelle de l'activité touristique est liée à la curiosité, au désir, de connaître l'autre, de ; satisfaire un besoin d'exploration et de vécus différents. Le tourisme n'existerait pas sans la culture ; la culture constitue l'une des principales motivations des déplacements des personnes aux musées et aux sites patrimoniaux. Toute forme de tourisme provoque un effet culturel aussi bien sur le visiteur (UNESCO, 2012).

Néanmoins, au fur et à mesure que le tourisme culturel se développe, les musées sont de plus en plus touchés. Le développement de ce type de tourisme au cours des dernières années a sans nul doute contribué à des changements d'importance dans les activités des musées. Le musée comme lieu de la pensée, de la réflexion, du plaisir et du savoir, se transforme en un processus socioculturel et économique aussi complexes que, notamment, la mondialisation, le

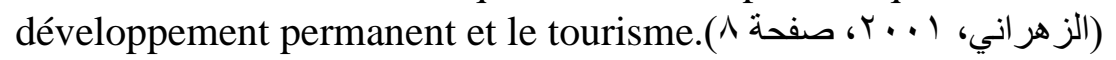

Une diversité ainsi induite dans toutes actions, de leur fonctionnement, de leur planification et de leurs relations avec le public. Les directeurs des musées deviennent plus que des administrateurs ; ils fixent les objectifs, dirigent les personnels. Cependant avoir à la fois la vision, la direction et la capacité de discerner les priorités (Porier , 1998, p. 70) .

\section{Tourisme culturel et patrimonial à Alexandrie}

Le tourisme culturel, apparait comme une forme nouvelle de tourisme spécialisé qui considère le patrimoine comme une fin en soi et suppose une harmonie fondée sur le respect des différentes cultures, sur la participation des communautés concernées et sur une juste répartition des bénéfices(El Wakil, La promotion du tourisme patrimonial en Egypte et les pays Africains:Vision pour un échange culturel et un développement touristique durable, 2016, p. 25).Le musée est moyen de découverte du visiteur et d'appréhension de toute activité culturelle, du fait que le nombre de musées égyptiens a atteint 77 à la fin de l'année 2019, dont 39 musées archéologiques, historiques et régionaux en 2019, contre 34 musées en 2018, d'une augmentation de $14,7 \%$. Les musées du Caire représentent 31,2\% du nombre total de musées, suivis du gouvernorat de Gizeh de 16,9\%, et Alexandrie de 10,4\%, selon les statistiques officielles publiées par l'Agence Centrale pour la Mobilisation publique et des Statistiques En raison du potentiel touristique, culturel et archéologique d'Alexandrie, le tourisme culturel est un facteur majeur de développement économique et culturel de la ville .

Le gouvernorat attire $36 \%$ du tourisme intérieur du pays. L'Autorité régionale pour la promotion du tourisme d'Alexandrie, a mentionné le nombre des visiteurs des sites, musées et la Bibliothèque d'Alexandrie de 2,64 millions de personnes en 2019. 1,14 million de visiteurs dont $71 \%$ d'Égyptiens, contre $29 \%$ d'étrangers, une augmentation de fréquentation des sites 
archéologiques et des musées par rapport à l'an 2018 de 992000 visiteurs et 2017 de 984000 visiteurs. Tandis que le nombre de visiteurs de la Bibliothèque d'Alexandrie a atteint environ

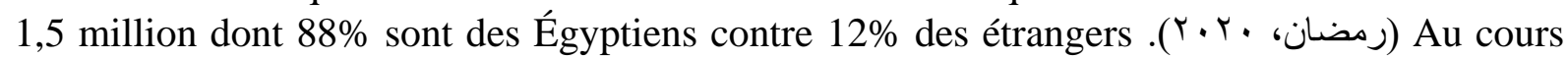
des mois de septembre et octobre les sites et musées d'Alexandrie ont accueilli 180427 visiteurs

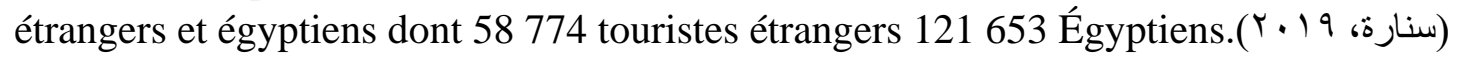

\section{Musées de la ville, mémoire du patrimoine alexandrin}

Sur la terre d'Alexandrie, Ptolémée 1er a fondé l'un des musées antiques par en 290 av. J.C, sur le même modèle que les écoles philosophiques d'Athènes, en particulier l'Académie de

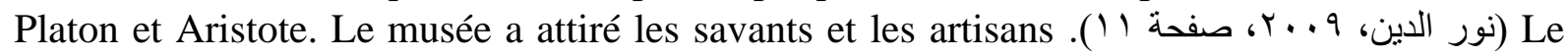
musée est un terme dérivé du mot grec Mouseîon dont le sens est temple, ou sanctuaire, c'est un lieu où sont réunies des collections en vue de leur conservation et de leur présentation au public (Le Larousse, 2015).Une reformulation de la fonction du musée a eu lieu, des idées et des capacités matérielles ont émergé soulignant le rôle culturel, patrimonial et économique des musées par la définition du Conseil International des Musées (ICOM) comme une institution permanente, sans but lucratif, au service de la société et de son développement (ICOM, 2011). Les musées traversent sans peine les barrières culturelles et linguistiques de point qu'ils doivent éveiller le public à des cultures différentes. L'éducation culturelle est la mission essentielle des musées, elle ne se limite à des références à une culture historique savante, certes, mais englobe, au contraire, les différents contextes géographiques, économiques, scientifiques et techniques, esthétiques... etc. dans lesquels elle s'inscrit. Elle est une partie intégrante d'une formation fondamentale, équilibrée et qui ne saurait être uniquement cérébrale ou conceptuelle (Allard, 1997). Contribuant à stimuler les besoins d'expressions et d'échange, le permet, par la découverte et l'éducation du goût de chacun, une affirmation plus libre et plus complète de la personnalité de tout individu. C'est l'une des voies de l'épanouissement des esprits (Forest, 2008). Bien entendu, Alexandrie bénéficie de la présence de plusieurs types de musées qui brisent l'identité et la personnalité alexandrine, au service du tourisme culturel et patrimonial, ces espaces ne doivent non seulement attirer les visiteurs, mais aussi les retenir par la médiation afin de stimuler leur impact social par l'apprentissage culturel.

Musée National d'Alexandrie, situé au cœur d'Alexandrie, classifié comme musée régional, est l'un des chefs-d'œuvre architecturaux uniques construit au style italien ( فزارة السياحة و الاثار Y. V V المصرية، تفاصيل المتاحف:متحف الاسكندرية القومي، Le bâtiment du musée était à l'origine le palais d'Asaad Bassili Pacha, jusqu'à 1954, puis il a été acheté par le consulat américain, en 1996. Le Conseil suprême des antiquités l'a acheté, l'a été restauré et inauguré en tant que musée en septembre 2003. Il se compose de trois étages comprenant plus de 1800 artefacts couvrant la plupart des étapes historiques de la civilisation égyptienne, de l'Ancien Empire à l'ère moderne. Le musée se distingue également par son exposition d'une collection unique d'antiquités englouties qui ont été découvertes lors de fouilles effectuées dans la baie d'AbouQuir à Alexandrie.

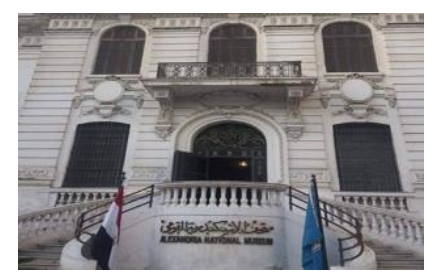

Figure 1 : Photo du Musée National, prise par la chercheure 
Musée des Bijoux Royaux, situé au quartier Zizinia est l'un des chefs-d'œuvre architecturaux uniques construits au style européen. Le bâtiment était à l'origine le palais de la noble de la famille royale alaouite Fatima Al-Zahra. Il se compose de deux ailes, est et ouest reliées par un couloir, et entouré d'un jardin. En 1986, le palais est devenu un musée contenant une grande collection de 11000 objets sélectionnés de bijoux royaux et d'objets rares de la famille royale, réparties en dix salles, tels que des décorations, des médailles, des colliers en or, des montres, des ensembles pour fumeurs et des ensembles décoratifs pour les reines et les princesses Fait d'or et de platine, parsemé de pierres précieuses et semi-précieuses, couronnes parsemées de

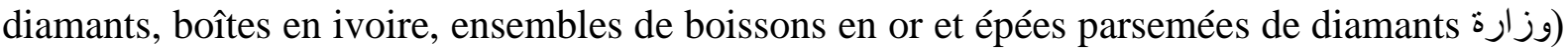

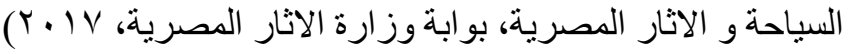

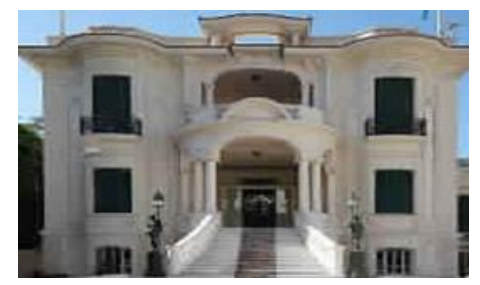

Figure 2 : Photo du Musée des Bijoux Royales, prise le 13/08/2020 du portail numérique du Gouvernorat d'Alexandrie sur:

http://www.alexandria.gov.eg/services/tourism/alextourism/museums/jewlerymusuem.html

Musées de la Bibliothèque d'Alexandrie, ce complexe culturel abrite quatre musées : " Le Musée Sadate » est le 1er en son genre dans la ville sur une surface de $260 \mathrm{~m} 2$, pour documenter une partie de l'Histoire de l'Égypte moderne. «Le Musée des Manuscrits » ayant un caractère spécial qui a pour vocation de faire connaître au public les œuvres patrimoniales, les manuscrits et les livres rares (El Wakil, Vers une éducation patrimoniale au service d'un tourisme durable à Alexandrie, 2017, pp. 223,224). "Le Musée des Antiquités » est l'un des rares musées au monde qui expose des artefacts découverts au même endroit où ils sont exposés. Il a été officiellement inauguré en 2002. Ses collections contiennent 1133 pièces soigneusement choisies dont la plus importante est un groupe d'artefacts découverts lors des travaux de fouille sur le site de la bibliothèque de 1993 à 1995, et un groupe d'antiquités qui ont été soulevées du sol méditerranéen près du port oriental et dans la région de la baie d'Abou - Quir (وزارة السياحة

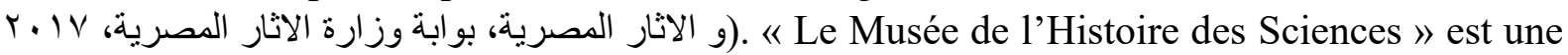
exposition permanente sur les exploits des Anciens Égyptiens durant trois époques majeures: l'Égypte pharaonique, Alexandrie hellénistique et le Moyen Age arabo-musulman. Il rend hommage aux grands savants ayant enrichi la connaissance humaine en faisant renaître leurs grandes découverte (Bibliotheca Alexandrina, 2015)

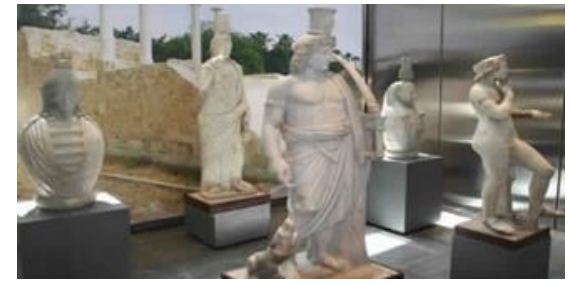

Figure 3 : Collections au Musée des Antiquités de la BA. Photo prise par la chercheure

Musée et Aquarium des Sciences Marines, situé au quartier Al-Anfouchi, à côté de la citadelle de Qaitbay, a été créé en 1930. Il comprend une collection de poissons rares avec des informations sur chaque espèce. Il se compose de deux parties. La première contient de grands groupes de poissons de diverses espèces dans des étangs fermés, et la seconde est ouverte et contient des structures momifiées et des fossiles pour les organismes aquatiques. Quant à l'autre moitié elle comprend des maquettes, des créatures vivantes et des dioramas mettant en valeur

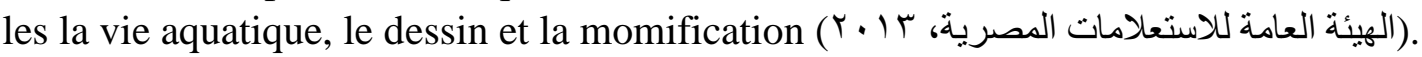




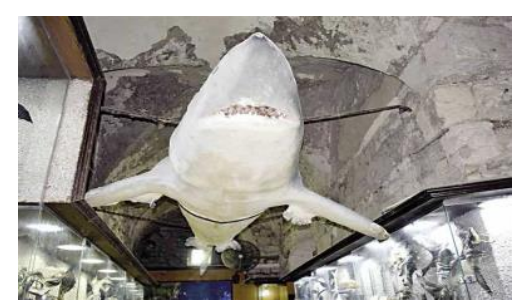

Figure 4 : Écuries marines au Musée Marin. Photo prise par Mahmoud Taha au Journal El Masry El Youm. N. issu le 29/01/2018

Musée des Beaux-Arts d'Alexandrie, situé à Moharam-Bék, il fut inauguré en 1954 comme le premier en Égypte et au Moyen-Orient spécifiée en Beaux-Arts. Il comprend une grande collection d'œuvres d'artistes égyptiens, en plus de quelques œuvres de l'école romantique, baroque et rococo, et quelques peintures orientalistes. Il comprend également quelques gravures

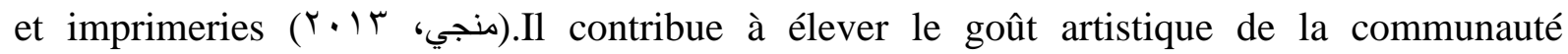
alexandrine à travers la mise en place de diverses activités artistiques tels que les ateliers artistiques, l'éducation muséale pour les enfants, et de nombreuses expositions d'art des activités d'échange culturel international.

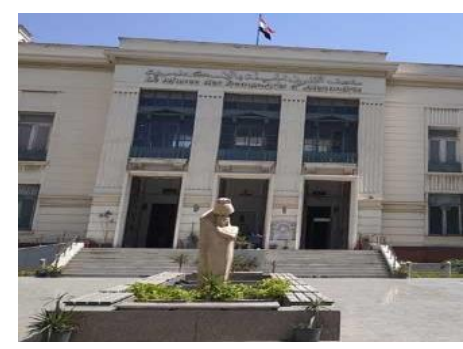

Figure 5 : Photo du Musée des Beaux-Arts, prise par la chercheure

Complexe muséal de Mahmoud Saïd, situé au quartier Ganaklis, inauguré en 2000, il comprend trois musées d'Art, dont le premier est le musée Mahmoud Saïd, l'un des pionniers de l'art plastique à l'époque moderne. Le deuxième au second étage est celui des deux frères Adham et Saif Wanli. Quant au troisième, situé au sous-sol du palais, il s'agit d'un musée d'art égyptien moderne d'Alexandrie (الهيئة العامة لتنشيط السياحة، 1999). Le site offre une vue panoramique sur les différentes salles d'exposition des trois musées, ainsi que sur les expositions et séminaires organisés au centre. Il présente également l'histoire du musée et des artistes et les dates importantes de leur vie à travers leur influence sur le mouvement artistique et présente des exemples de leurs œuvres.

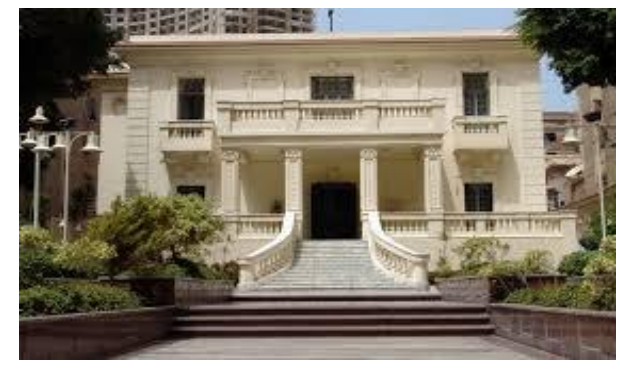

Figure 6: Photo du Musée de Mahmoud Saïd, prise le 17/08/2020 du Site Officiel du Ministère de la Culture sur http://www.moc.gov.eg/ar/affiliates$\underline{\text { list }}$

Musée de Cavafy, au centre-ville fut inauguré en 1992 lorsque Kostis Moskov, le conseiller culturel grec, vint à Alexandrie et transforma la pension en musée affilié à l'ambassade de Grèce en Égypte afin de commémorer le grand poète grec Constantin Cavafy l'un des poètes les plus célèbres de l'ère moderne. La maison a une grande valeur archéologique, elle a été transformée en pension sous le nom de Pension Amir en 1958 le resta pendant 33 ans jusqu'en 1991 par sa transformation en musée. La société grecque à Alexandrie a transféré les biens du

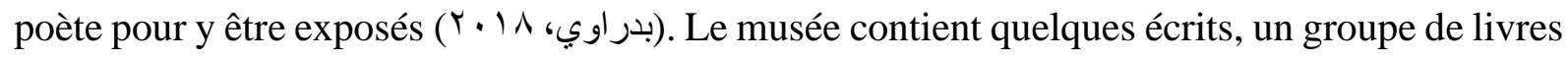
internationaux en 70 langues étrangères, de diverses photos personnelles des souvenirs et des 
outils personnels. Les expositions comprennent également une grande collection de vidéos sur le poète, sa biographie, sa vie et ses œuvres.

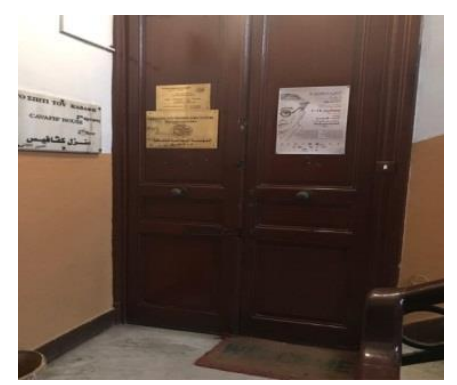

Figure 7 : Photo du Musée Cavafy, prise le 20/08/2020 du site officiel de l'Administration Centrale du Tourisme et des Stations sur le lien: https://catr.gov.eg/almazarat

\section{Musées d'Alexandrie entre défis et possibilités}

Les musées sont divisés en trois sections principales : les Musées d'Art, spécialistes dans la présentation des réalisations artistiques de l'humanité, et comprennent les beaux-arts et les arts appliqués ; les Musées d'Histoire doués dans la présentation des réalisations humaines dans divers domaines au cours de l'histoire; et les Musées Scientifiques, divisés en deux types: Le premier des sciences naturelles, comme la physique, la chimie et les mathématiques et le deuxième type, englobant les musées d'histoire naturelle des plantes, des animaux et de la géologie , نور الدين, 9.). (Les composants des musées varient en fonction de leur type et leur taille, généralement, ils contiennent des galeries et des salles d'exposition proportionnelles à la nature des collections. Des services liés aux salles tels que le service de maintenance, de stockage des matériaux, et des services techniques. Un service administratif de gestion globale du musée ; un service d'accueil et de sécurité ; un service culturel responsable des activités

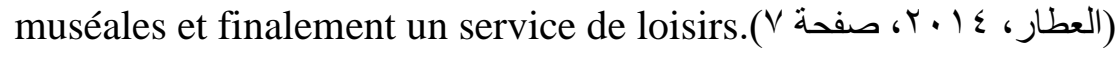

Les musées de la ville diversifient en nature de classification, de même leurs autorités de contrôle et de gestion, du fait qu'on observe une complexité au niveau de la politique culturelle muséale. La majorité des musées est établie dans d'anciens bâtiments, sans doute, les palais ajoutent une atmosphère historique et archéologique appropriée à la nature des collections ; ce qui développe le sens culturel des visiteurs. Mais, d'un autre part, l'exposition des collections doit respecter les conditions du bâtiment et l'impossibilité d'y apporter des modifications. L'application des méthodes muséales modernes est limitée, ainsi tout aménagement

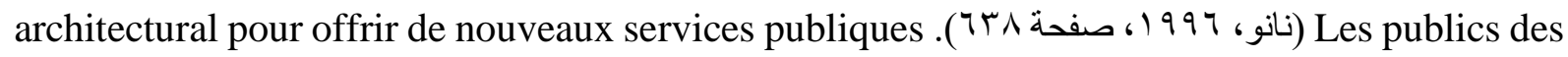
musées sont différents en fonction de leur niveau d'intérêt du fait que les stratégies de relations publiques varient, les gestionnaires traditionnels ont une orientation à sens unique. Cette vision ancienne ne s'intéresse plus à la fidélisation ou l'attraction du public (Gürel \& Kavak, 2010, pp. 54,57).En plus, on remarque l'absence d'un service spécialiste de relations publiques, les taches sont interconnectés, les personnels d'accueil sont même les agents de sécurité, un déficit de la spécification des missions. Ces difficultés au niveau d'entretien, de renouvellement et de communication nécessitent des efforts particuliers pour devenir aptes aux nouvelles demandes. Cette complexité exige que les musées promeuvent leur travail comme service, cependant, peu de recherches scientifiques portent spécifiquement sur les relations publiques dans les musées et l'exigence de ce service de communication pour nourrir le dialogue entre le musée et ses visiteurs; en effet, la survie du musée dépend du public. 


\section{Service des Relations Publiques aux musées}

Au sens général, le service des relations publiques établit une compréhension mutuelle entre l'organisation et ses publics et consiste à gérer la communication entre eux (Harrison, 1995). Néanmoins, il n'existe pas de définition formelle des relations publiques dans les musées; mais on peut la définir du point qu'on considère le musée comme un lieu ayant un statut institutionnel, d'une motivation organisationnelle accueillant publique multiple; comme un secteur au service des visiteurs de tous publics, il s'intéresse à leur opinion pour mieux comprendre leurs besoins. Les relations publiques au musée est un domaine relativement nouveau en muséologie, il carde l'ensemble de normes et d'objectifs de communication dans la mesure d'améliorer l'expérience des visiteurs (Black, 2011). C'est pourquoi les musées sont de plus en plus engagés et deviennent plus interactif. Avoir des services de relations publiques performants dans les musées ajouterait également de la valeur et des informations, cela aiderait également à identifier les objectifs communs et les stratégies utilisées au sens large. Dans l'ensemble, les relations publiques des musées alexandrins ont encore un long chemin à parcourir.

\section{Étude de cas}

Pour évaluer efficacement l'État actuel des relations publiques aux musées, on a formé un questionnaire qui mesure l'aperçu des visiteurs autour de leur visite, des services et des activités sur place. On a travaillé sur un échantillon des visiteurs existants sur place à Alexandrie au mois de janvier 2020.Une méthode scientifique qui a mené à définir l'unité de sondage, la taille de l'échantillon et la procédure de tirage. La sélection de l'échantillon est effectuée de façon aléatoire pour appliquer les règles de l'inférence statistique. La taille de l'échantillon était de 130 visiteurs, 7 questionnaires ont été éliminés car ils ne répondaient pas aux critères. On a voulu par ce sondage de faire sortir des résultats pour atteindre une représentativité qui pourra être généralisée à tous les visiteurs des musées. Le questionnaire mesure le degré de la communication, de relation interactive mutuelle entre le musée et les visiteurs; la qualité des services et activités offertes pour mesurer l'efficacité des relations publiques aux musées qui influencent sans doute la satisfaction du public. Les questions portaient en première partie sur les visiteurs et les antécédents de leur visite, puis en une seconde partie, on les interrogeait autour les sources d'informations portant sur les musées, leurs motivations et attentes de la visite muséale pour en finir par la troisième section à savoir leurs intérêts aux sections muséales, le niveau d'appréciation de la visite, de satisfaction et la disposition à revenir et à revisiter le musée.

\section{Types de visiteurs et compagnie}

L'analyse statistique nous offre une exactitude de données, un taux de $27 \%$ pour les visiteurs étrangers et de $73 \%$ pour les Égyptiens. Cela montre la fréquentation locale, que ce soit de l'intérieur ou de l'extérieur du gouvernorat, et que la majorité des visiteurs des musées, tout au long de l'année sont des Égyptiens et non des étrangers. En plus la majorité du public vient en groupe d'un taux de $63 \%$ contre $37 \%$ pour les visiteurs individuels, une indication que la plupart des visites est sous la forme des excursions. 
—Égyptiens घÉtrangers

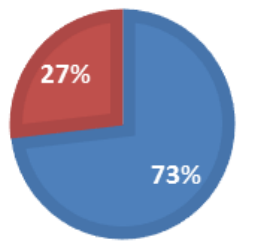

- Seul En Groupe

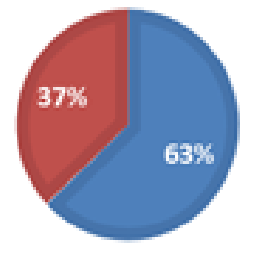

Fiaure 9: Compaanie du visiteur

\section{Nombre, but, et durée de la visite}

Sans doute, la nature et le but de la visite diffèrent d'une personne à une autre, les participants ont montré que $66 \%$ de l'échantillon fréquentait les musées en tant que touriste égyptien ou étranger, $18 \%$ à des fins éducatifs et $16 \%$ pour jouir d'autres activités muséales. Quant au nombre de visite, $78 \%$ ont déclaré que c'était leur première contre $22 \%$ qui ont indiqué une répétition de l'expérience muséale. La durée de la visite pour la plupart de $72 \%$ ne dépasse pas une heure et la minorité d'un taux de $28 \%$ pouvait y rester pour plus d'une heure. Il existe une relation directe entre le but et la durée de la visite, ils augmentent ou diminuent conjointement, en plus, plus le visiteur est attiré par le musée plus le nombre de ses visites augmentent et vice versa.

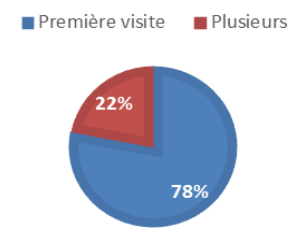

Figure 11 : But de la visite

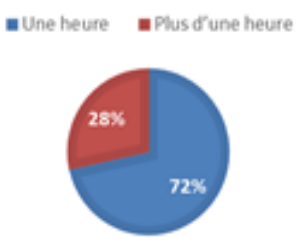

Figure 9 : Nombre de visites du musée

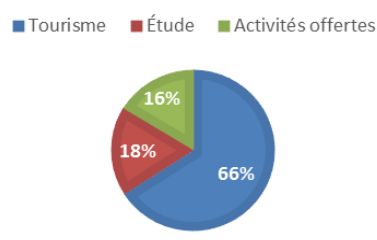

Figure 10 : Durée de la visite

\section{Informations sur le musée et ses manifestations}

Les informations sur le secteur muséal et les potentiels touristiques d'Alexandrie sont insuffisantes pour développer adéquatement le tourisme culturel et la fréquentation muséale, parmi les répondants à l'enquête, 55\% étaient d'avis qu'il y avait suffisamment d'informations disponibles sur les musées. Cependant, un bon nombre de répondants de $45 \%$ estimaient que les informations n'étaient pas facilement disponibles. Cela suggère qu'il y a encore des problèmes de disponibilité des informations surtout après leur signalisation que la source principale était l'internet de $48 \%$ suivi par les guides et les livres et finalement la source de bouche à oreille de $22 \%$.

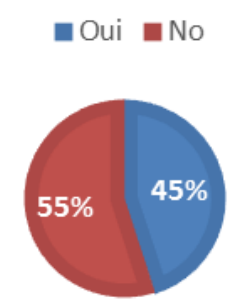

Figure 12 : Informations sur le musée et ses manifestations

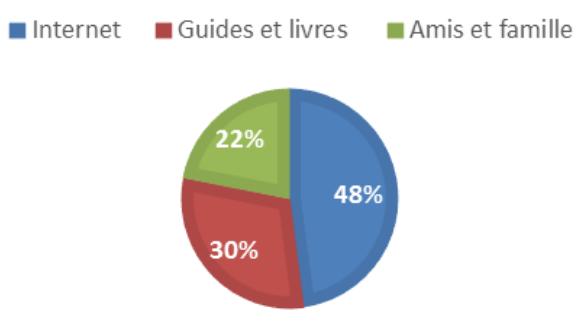

Figure 13 : Sources d'informations 


\section{Intérêts aux sections muséales}

L'attention est le principal moteur de tout, plus la direction muséale est consciente de l'importance de la diversité de l'offre culturel, plus le musée est en mesure d'attirer le public et plus son efficacité économique accroit.Les musées Alexandrins ont été créés pour recevoir une collection existante comme base, ils travaillenttraditionnellement, les expositions sont permanentes et immobiles. Les activités muséales viennent de commencer récemment, elles souffrent de manque de propagande, de promotion et de fonds pour attirer les gens. Les participants annocaient leur interet aux collections d'un taux de $75 \%$ contre $25 \%$ d'eux pour les activités, soit en raison de la manque d'activités ou à cause de leur ignorance autour de la vie culturelle au musée.

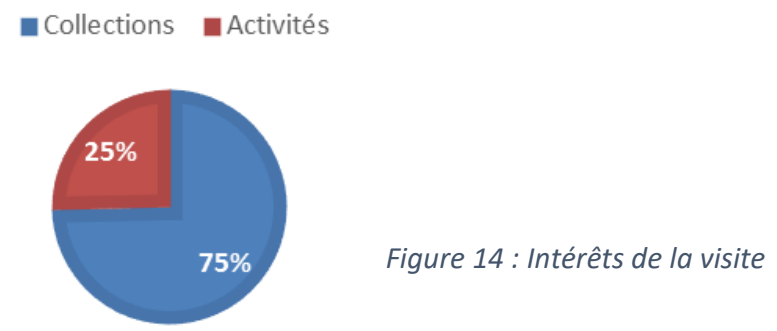

\section{Appréciation de la visite}

Il est très important de connaître l'impression du visiteur, car d'une part il se sent important pour l'organisation et d'autre part, son opinion contribue à rendre le musée plus apte à se développer pour satisfaire les motivations de ses visiteurs. On a mesuré le degré d'appréciation des visiteurs sur divers aspects de la visite (l'accueil, les informations, la signalisation, les services et les relations publiques). Le choix est personnel, il montre le degré d'appréciation du participant par les niveaux (élevé, moyen ou médiocre). La figure ci-dessous, indique une approbation générale pour le niveau médiocre pour tous les aspects, même si les proportions diffèrent (68\% pour l'accueil, 63\% pour les informations, $73 \%$ pour la signalisation, $64 \%$ pour les services et $92 \%$ pour les relations publiques).Ces faibles indicateurs indiquent la nécessité de la redéfinition des tâches et la nécessité de tracer une nouvelle politique muséale centrée sur le visiteur et la création d'une section spéciale pour les relations publiques qui fera le lien entre l'institution et son visiteur. En deuxième rang, venait le choix du niveau moyen et finalement le niveau élevé comme suit $(35 \%$ contre $20 \%$ pour 1 'accueil, $38 \%$ contre $22 \%$ pour les informations, $32 \%$ contre $18 \%$ pour la signalisation, $37 \%$ contre $22 \%$ pour les services et $18 \%$ contre $13 \%$ pour les relations publiques.)

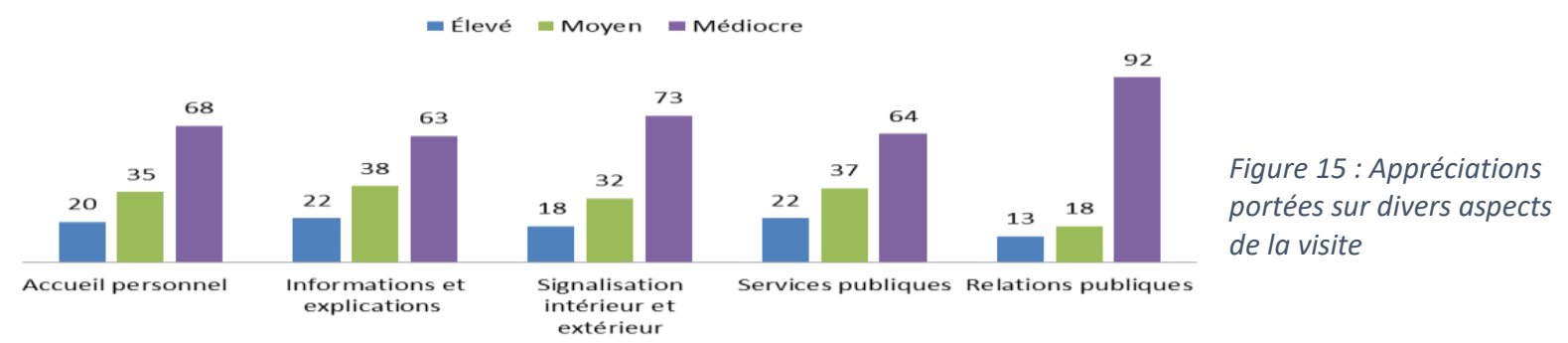

\section{Disposition à revenir}

La répétition de la visite est l'une des principales cibles des gestionnaires des musées. Motiver les visiteurs à revenir et attirer de nouveaux publics sont des buts réalisables par la promotion des prix, de l'offre et la motivation liée aux caractéristiques du musée visité. Le résultat de cette rubrique montrait un grand écart entre les résultats des deux indicateurs, $78 \%$ contre $22 \%$ des participants ont exprimé leur indisposition de se rendre à nouveau au musée, cette réticence 
peut découler soit de la dimension spatiale, soit de l'absence des facteurs d'attraction qui les incitent à renouveler la visite.

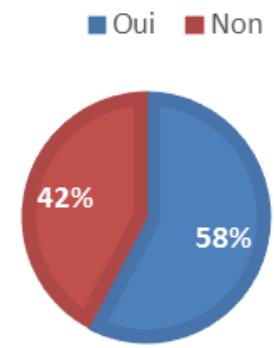

Figure 16 : Dispositions à revenir

\section{Stratégie muséale recommandée}

Aujourd'hui, la planification stratégique dans le secteur du tourisme en général et des musées en spéciale est considérée comme l'un des moyens qui contribuent à l'avancement et à la revitalisation du secteur. Un processus pratique dirigé et un style de travail organisé pour réaliser l'investissement optimal des ressources disponibles pour atteindre des objectifs clairs et spécifiques(صدار و زدايرية، 7 ( • ץ، صفحة 7 Le cadre général de la nouvelle stratégie du secteur des musées élaborée par le Ministère de la Culture en 2019 vient pour être en coordination avec

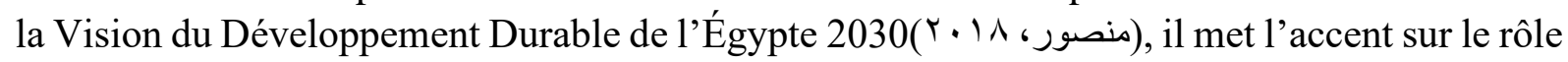
culturel, social et économique des musées. Sur la base de ces principes, on propose la stratégie suivante recommandée pour un meilleur avancement des musées Alexandrins.
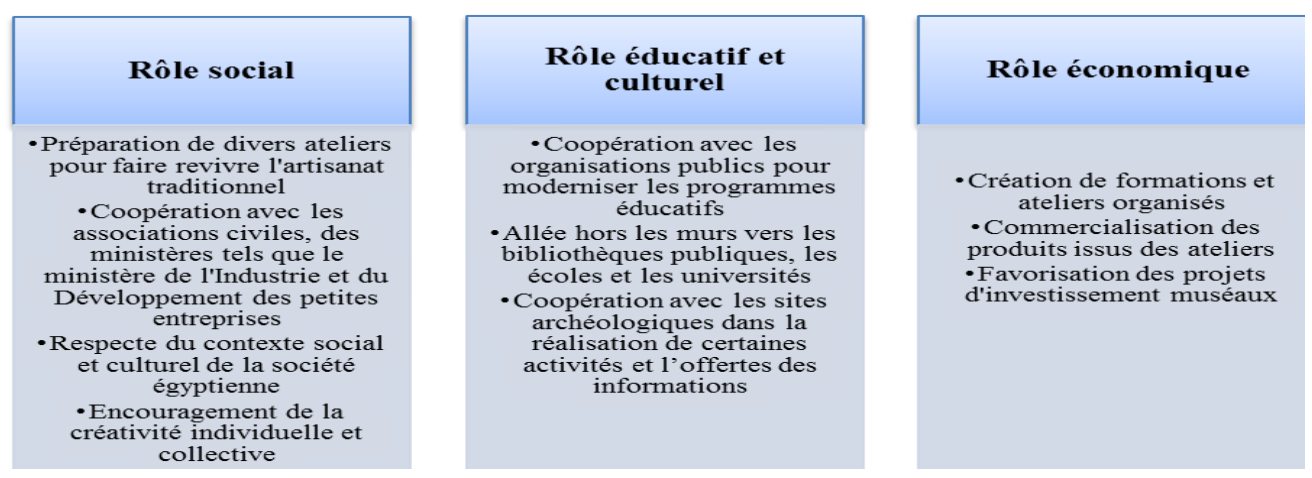

Figure 17: Cadre général de la Stratégie muséale, élaboré par la chercheure. Informations tirées et traduites du Site Web officiel du Ministère de la Culture le 27 août 2020

La stratégie proposée est une approche générale sous la forme d'un plan d'actions non détaillé, conçu pour atteindre les objectifs culturels visés. La figure(18) est considérée comme une carte que les musées s'engagent à suivre pour les rendre plus dynamique en vue de la fréquentation du public culturel. Elle est ciblée, évaluative et coordonne avec la Stratégie du développement du Ministère de la Culture et du Pays dans le choix des priorités. Une fois le plan d'action sera mis en place tous les acteurs et les parties prenantes sont priés à interagir par leurs expertises.

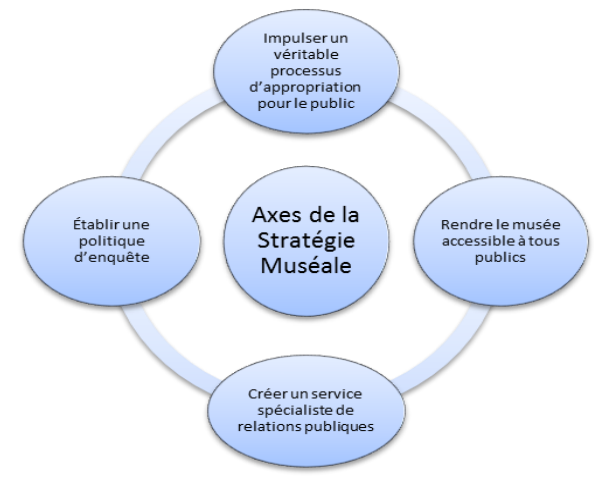




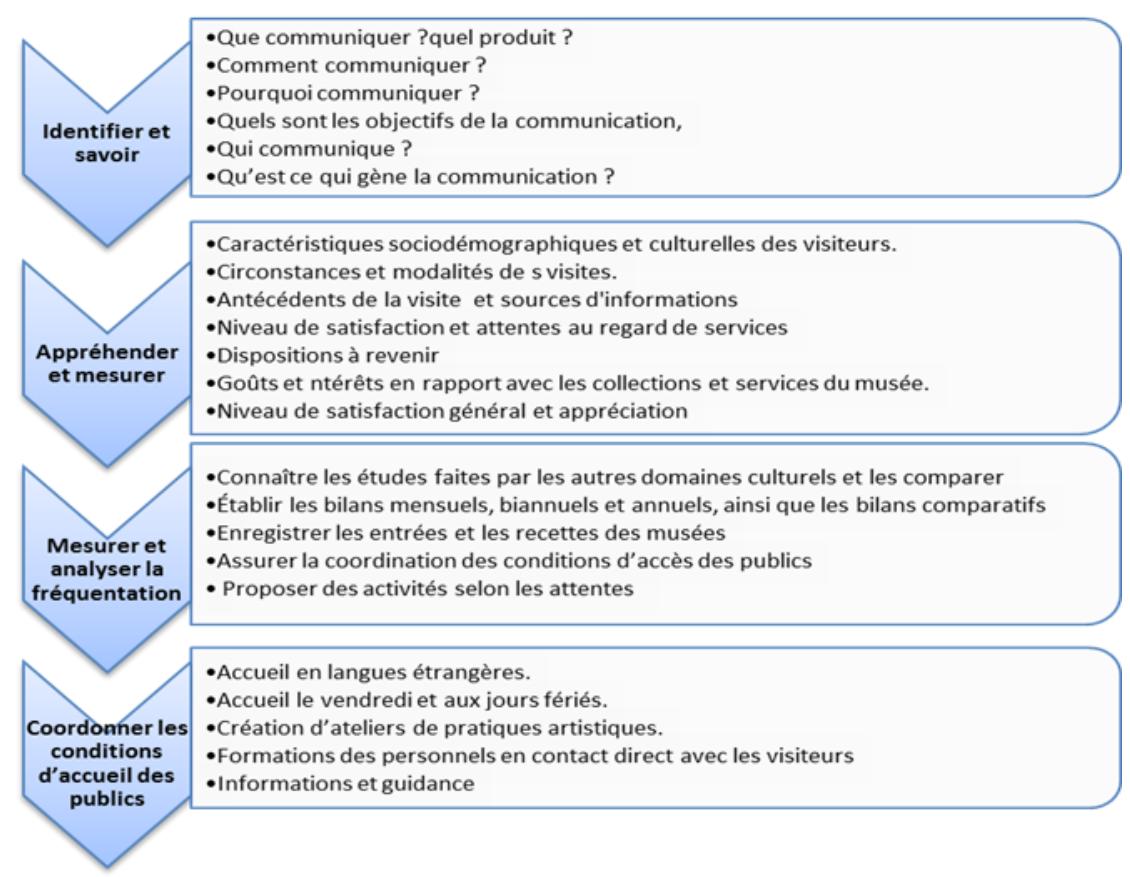

Figure 19 : Missions visées du Secteur des Relations Publiques aux Musées, préparées par la chercheure

Établir une politique d'enquête: Les études clients appelées aussi études sur les visiteurs constituent un domaine spécialisé qui renseigne sur les visiteurs et autres bénéficiaires des attractions patrimoniales. Elles visent à déterminer qui sont les visiteurs, quels sont leurs motifs de visite, leurs attitudes, leurs attentes, leurs sources de satisfaction et de mécontentement ( (المؤسسة العامة للتعليم الفني و التندريب المهني، ع. . Elles sont mal connues aux musées égyptiens. Les études clients jouent un rôle fondamental dans la planification générale et globale ainsi que dans la prestation d'un service efficace au public. Elles aident les professionnels du patrimoine culturel dans des domaines de planification stratégique, de prospection de la clientèle, du

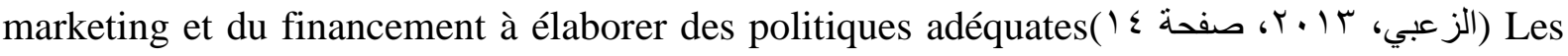
objectifs visés de cette politique est de fournir des données objectives à des fins décisionnelles, d'éviter de recourir à des hypothèses théoriques et inexactes relativement aux publics et de réduire les erreurs et maximiser le succès au niveau de la planification (El Wakil, Mémoire de DEPA: Musée Gréco-Romain d'Alexandrie:Comment améliorer le service des publics pour mieux répondre aux éxigences du tourisme culturel, 2005, p. 96).

\section{Rendre le musée accessible à tous publics}

- Nouvelle image muséographique

Le musée doit travailler l'image qu'il donne aux visiteurs dès l'entrée, il faut d'abord qu'il connaisse ses publics, qu'il définisse ensuite sa stratégie. Le musée doit prendre en compte ses facteurs car il est toujours présent en tant que médium offrant la collection au public. Plus il devient un véritable lieu de rencontre, plus il sera vécu comme un média et plus son environnement sera la condition de toute communication avec la collection.

Tout musée est d'abord vécu comme un entrepôt renfermant une accumulation d'objets. Les visiteurs aiment être orientés sans se perdre, pour composer leur propre visite. Les panneaux et les notices s'adressent aux publics explorateurs, d'où l'importance de proposer un ordre d'une visite organisée, fournir une documentation et un nécessaire complément d'information aux visiteurs (حسين, 2006, pp. 119,125).

- Médiation entre visiteurs et collections 
La médiation culturelle consiste à créer un rôle intermédiaire entre ceux qui détiennent un savoir, et le public dont il faut prendre en compte ses habitudes, ses attentes et ses aspirations. Le musée est un lieu de médiation culturelle qui consiste à un va et vient entre ceux qui savent et ceux qui ne savent pas. Une action qui montre, explique, et situe les objets dans leur contexte afin de déchiffrer ces objets et les ressentir (Tarpin, 1995). L'essentiel demeure l'accompagnement personnel, vivant, au cours duquel établir un dialogue qui sert à découvrir la diversité des formes et des expériences, et d'entraîner la mobilité intellectuelle et affective (Allard, 1997).

\section{- Renforcer les politiques éducatives culturelles}

Le musée est une institution destinée à satisfaire les préoccupations d'adultes et d'enfants. Dans la même mesure où ils doivent s'intégrer à la culture de la société au sein de laquelle ils sont nés ( 9 (غنيمة، .99). Il s'agit de les inciter à voir les objets dans leur réalité, et ensuite, chaque fois que cela est possible, à les toucher ou à les manipuler (Roland, 1994).

- Élargissement de la fréquentation

Il est nécessaire, en premier lieu, de connaître exactement la fréquentation effective du musée, au moyen de statistiques, de sondages, d'enquêtes qualitatives. Il est important, à partir de ces données de distinguer les motivations diverses des visiteurs. Il est capital d'analyser les différents freins qui peuvent empêcher ce public de fréquenter le musée.

Il est ensuite nécessaire de tout faire pour tenter de mettre le visiteur inhabituel en attitude active. Cela est réalisable par le recours à des thèmes d'intérêts généraux et actuels, par les

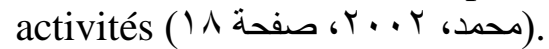

D'une façon générale, la stratégie en vue de l'élargissement des publics surtout les locaux suppose l'élaboration explicite d'une politique des publics, définissant des alliances et des partenariats possibles avec des organismes divers et en proposant des systèmes d'adhésion.

- Programmation des expositions

Une collection meurt si on ne continue pas à l'enrichir et la mettre en valeur. L'exposition et le musée sont devenus depuis une dizaine d'années un phénomène social et un objet esthétique. L'exposition est un moyen efficace de communication, un événement culturel offert au public qui exige des connaissances spécialisées ( Ministère de la Culture, 1999). Le Service pourra organiser une sorte d'exposition temporaire comme un nouvel mode de communication particulier, qui contribue à la vie et l'animation du musée; elle utilise ses multiples données potentielles et restitue au public des objets et les abordent selon des points de vue différentes ce qui provoque l'échange et la connaissance (199. غنيمة، (19).

\section{Impulser un véritable processus d'appropriation pour le public}

- Accueil et confort du visiteur

La visite du musée doit être vue comme un élément de plaisir et convivialité pour le visiteur : de l'accueillir véritablement et de rendre sa visite la plus agréable possible. Il faut envisager tous les aspects contribuant à créer l'atmosphère idéale à son confort. Celui-ci est à la fois physique et psychologique et intellectuel. Il implique toute une série de services et d'aides indispensables, tant à l'extérieur qu'à l'intérieur du musée (Ministère de la Fonction publique, 1994). L'accueil est une sorte d'orientation, d'information, d'écoute, de service et d'assistance ce qui forment l'ensemble des demandes des publics. L'accueil c'est la réponse que doit y apporter naturellement un organisme public. Le contact avec le visiteur est la vitrine du service public il reflète l'organisation et le mode de management mis en place (El Wakil, Mémoire de DEPA: Musée Gréco-Romain d'Alexandrie:Comment améliorer le service des publics pour mieux répondre aux éxigences du tourisme culturel, 2005, p. 65).

L'améliorer est un gage de son efficacité ; mais cela suppose d'y consacrer beaucoup d'efforts de mieux comprendre les évolutions et les enjeux de la relation avec le visiteur, de mobiliser 
les collaborations du musée, de piloter une démarche globale d'amélioration des relations avec eux.

- Commercialisation des services publics

Le musée n'est plus seulement un lieu de délectation, ce n'est pas seulement un ensemble de salles de présentation d'œuvres, un espace d'accueil du public, c'est aussi tout un équipement avec des activités complémentaires. Le rôle et la place de chacune sont très importants, à cet égard une connaissance préalable du public visé est indispensable (Goad Blav , 1996).Avoir une démarche marketing valorise l'espace commercial muséal et évoque en aval une connaissance des demandes. La commercialisation des prestations, services et objets artisanaux est à la fois un art et une science. L'art de reconstituer une histoire et de la présenter dans le contexte du musée, cet environnement commercial exceptionnel. Savoir renseigner le client assure la continuité d'une tradition et favorise la relation entre les objets et les musées

\section{Conclusion}

En effet, la gestion des flux touristiques est de mieux en mieux maîtrisée par certains musées, techniquement parlant. Mais l'offre des musées d'Alexandrie vis à vis du tourisme culturel reste peu intégrée aux logiques nouvelles qu'impose ce tourisme. Les musées d'Alexandrie devront s'insérer dans un monde en mutation, adopter des méthodes de travail et une optique plus modernes qui leur permettront de trouver des formules inédites, plus proches, peut-être d'une stratégie de développement dans leurs secteurs. Il sera ainsi assuré de se procurer les ressources nécessaires à la poursuite de leur tâche, à la réalisation de leurs objectifs, à la sauvegarde des collections et la gestion des publics. Les musées doivent instaurer les meilleures relations et recherches possibles avec le tourisme pour acquérir une dimension nouvelle, d'agir comme institutions sociales qui non seulement conservent et protègent le patrimoine, mais également, le fait connaître aux populations autant qu'aux touristes pour leur intérêt et leur plaisir .

Le point de départ est un changement nécessaire des mentalités; les deux secteurs du tourisme et des musées doivent établir des relations qui tiennent compte de leurs particularités propres, et qui débouchent sur la formulation de propositions originales et véritablement novatrices reposant sur une excellente connaissance de la profession.

C'est au professionnel des musées qu'il appartient de concevoir le touriste comme un allié qui doit être conquis pour assurer la préservation et la jouissance des biens culturels, ainsi que de favoriser le dialogue interculturel.

Ce changement d'attitudes repose sur l'idée que le musée désormais être tenu pour un moyen de communication entre le patrimoine et le public par l'intermédiaire de l'objet, au lieu d'être uniquement conçu comme un sarcophage d'objets fameux à tous jamais sacralisés et momifiés. Les muséologues doivent donner au musée une souplesse et un esprit d'entreprise, de mettre au pied des services nouveaux, en accord avec l'évolution des besoins des musées et de leurs usages. Si le musée est considéré comme un instrument de communication, non pas uniquement comme une source d'informations, mais comme un élément d'interaction entre le public et les phénomènes et produits culturels, la force d'un langage peut être mise à profit, un langage qui n'est pas verbal, mais qui se fonde sur l'articulation d'objet, qui suscite des idées et des émotions chargées de valeurs et de contenus susceptibles d'être appréhendés et assimilés par le public. 


\section{BIBLIOGRAPHIE}

(n.d.). Accés en octobre 25, 2017, sur www.lemonde.fr/voyage/article/2009/03/01/alexandriecite-antique 25/10/2017.

(n.d.). Accés en mai 23, 2018, sur https://www.bibalex.org/fr/page/museums 23/5/2018.

Ministère de la Culture. (1999). Musées et Service des Publics. Paris, France: Direction des musées de France..

$(Y \cdot I V)$ Accés en Octobre 25, 2017, sur: www.alexandria.gov.eg/alex/fr/index.aspx.

Allard, M. (1997). Le musée, un lieu éducatif. Montéréal, Canada: Musée d'art contemporain de Montréal.

Bibliotheca Alexandrina. (2015). BA museums. Accés en mai 23, 2018, sur Bibliotheca Alexandrina: https://www.bibalex.org/en/page/museums

Black , J. (2011). Master of Arts in Public Communication:Museum public relations: Investigating the role of PR in the museums.Washington: Americain University.

Claude, M. (2001). Alexandre : la destinée d'un mythe. Paris, France: Bibliothèque Payot et Rivage.

Conseil, R. (2016). Études sur les pratiques muséales en éducation, action culturelle et médiation. Québec: Société des musées de Québec.

Désbouis, G. (1991). Actes du colloque Mulhouse Ungersheim: répertoire analytique des musées, bilans et projets, 1980-1993. Acte du Colloque Mulhouse.. Mulhouse Ungersheim: Ministère de l'éducation nationale et de la culture, Direction des musées de France, Inspection générale des musées.

Direction des musées de France. (1994). Musées et tourisme. (S. d. France, Ed.) France: Ministère de la Culture.

El Wakil, R. (2005). Mémoire de DEPA: Musée Gréco-Romain d'Alexandrie:Comment améliorer le service des publics pour mieux répondre aux éxigences du tourisme culturel. Alexandrie, Égypte: Université Senghor.

El Wakil, R. (2016). La promotion du tourisme patrimonial en Égypte et les pays Africains:Vision pour un échange culturel et un développement touristique durable. Journal of Association of Arab Universities for Tourism and Hospitality, 25.

El Wakil, R. (2019). Vers une éducation patrimoniale au service d'un tourisme durable à Alexandrie. la Revue internationale du patrimoine, du tourisme et d'hospitalité , Faculté du tourisme et d'hôtellerie, Université de Fayoum, 4/2(5éme).

Empereur , J.-Y. (1998). Alexandrina1, 1998, pp 21-32. Le Caire, Égypte: Institut Français d'Archéologie Orientale (IFAO).

Empereur, J.-Y. (2001). Alexandrie: hier et demain. France: Découvertes Gallimard.

Errera, E., \& Mourrain , S. (2001). Le goût d'Alexandrie. Paris, France: Le Petit Mercure. 
Forest, M. (2008). Education et action culturelle: guide pratique. Quebec, Canada: Bibliothèque et archive nationale du Québec.

Goad Blav , D. (1996). Les Musées et le monde réel : le concept du musée. Muséum international(N.162).

Gürel, E., \& Kavak, B. (2010). A conceptual model for public relations in museums. European Journal of Marketing, 24(1/2), 42-65.

Harrison, S. (1995). Public relation:An introduction. London: Routledge.

ICOM. (2011). International Council of Museums. Retrieved April 05, 2020, from Museum Definition: https://icom.museum/en/resources/standards-guidelines/museum-definition/

Le Gouvernerat d'Alexandrie. (1989). L'histoire et la civilisation d'alexandrie à travers les âges. Alexandrie: l'Authorité Régionale pour le développement du tourisme.

Le Larousse. (2015). Le petit Larousse illustré. France: Larousse.

Ministère de la Fonction publique. (1994). Réussir la relation avec l'usager:Réflexion, méthodes et expériences pour améliorer l'accueil . (d. 1. Ministère de la fonction publique, Ed.) France.

Porier, J. (1998). Le tourisme (2) : le musée de la corse. Muséum international(200), 70.

Roland, M. (1994). Musées et éducation. Publics et Musées(N.7).

Tarpin, C. (1995). Thèse de doctorat en communication:Les musées québécois : de la sauvegarde de la mémoire collective à la communication. Montréal, Canada: Université Concordia.

UNESCO. (2012). Museums and heritage (Culture and developpement ed.). Madrid, spain: Embajada de Norwega.

$$
\begin{aligned}
& \text { المؤسسة العامة للتعليم الفني و التنديب المهني. (؟ . . ץ). اساسيات التسويق. المملكة العربية السعودية: الادارة العامة لنطوير } \\
& \text { المناهج }
\end{aligned}
$$

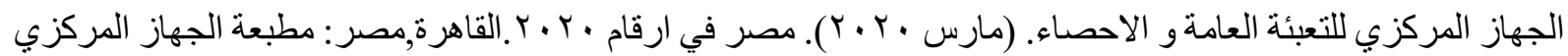

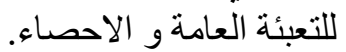

$$
\begin{aligned}
& \text { الزعبي, ع. (r ا • r). التسويق السياحي و الفندقى: مدخل صناعة السياحة و الضيافة.عمان: دار المسيرة للنشر و التوزيع و } \\
& \text { الطباعة. } \\
& \text { الز هر اني, ع. ( ا . . Y). السياحة التر اثية. السعودية: جامعة الملك سعود. } \\
& \text { الثيبانى, ع. (9V0 (190). مناهج البحث الاجتماعي.طر ابلس, ليبيا: الثركة العامة للنشر و التوزيع و الاعلان. }
\end{aligned}
$$

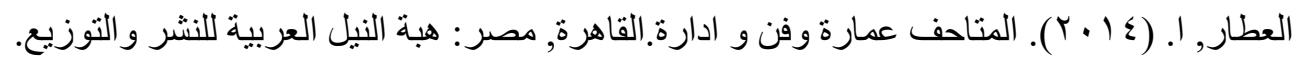

$$
\begin{aligned}
& \text { الهيئة العامة لتنشيط السياحة. (999 (199). دليل الاسكندرية. الاسكندرية, مصر : محافظة الاسكندرية. }
\end{aligned}
$$

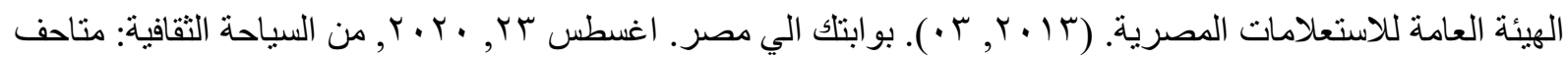

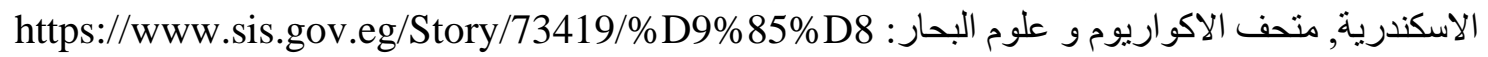

$$
\text { بدر , ا. (799 19). اصول ابحث العلمي و ناهجة(Vol. الطبعة الثنانية). الجيزة, مصر: المكتبة الاكاديمية. }
$$




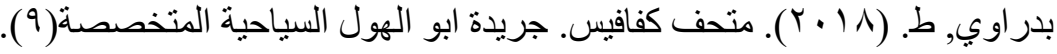

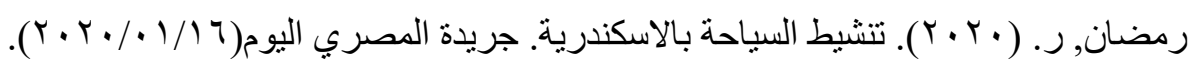
رياض, ه. (9Av 1 ). دليل آثار الأسكندرية .الإسكندرية: الهيئة الأقليمية لتنشيط السياحة.

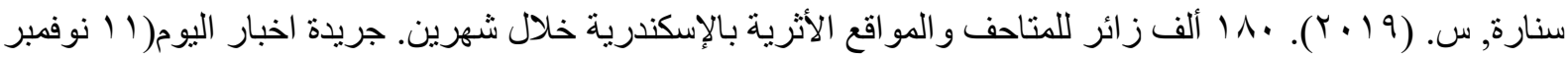
.$(Y \cdot 19$

صدار, ا., \&زدايرية, ر. (7 أب). رسالة ماستر اكاديمي بعنوان: دور التخطيط الاستراتيجي في تحقيق تنمية سياحية

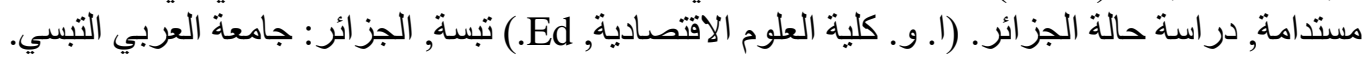

غنيمة, ع. (• (99 (). المتاحف, لمعارض و القصور وسائل تعليمية.المنوفية, مصر: سلسلة المعرفة الحضارية.

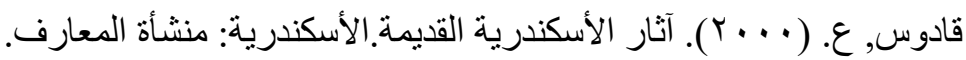

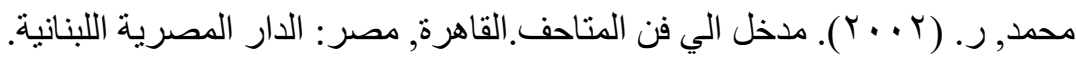

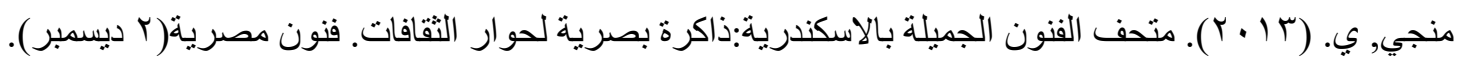

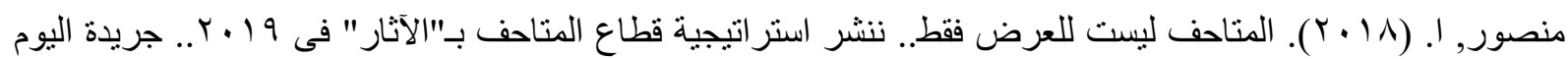

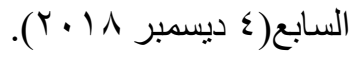

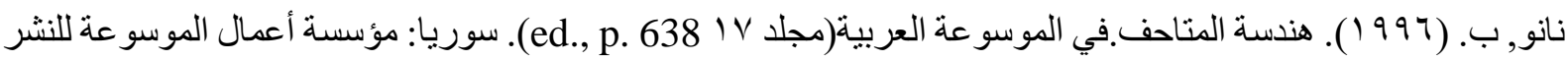
والتوزيع.

نور الدين, ع. (9 . r). متاحف الاثار في مصر و الوطن العربي.القاهرة, مصر : لأقصى للطباعة.

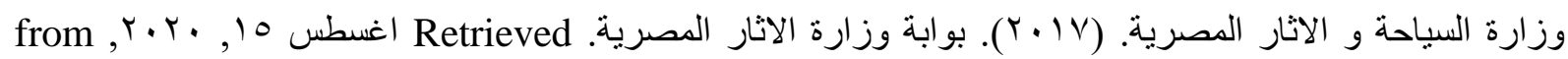

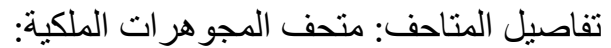

http://www.antiquities.gov.eg/DefaultAr/Museum/Pages/MuseumDetails.aspx?MusCode=16

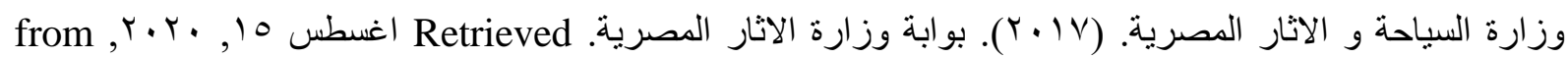
تفاصيل المتاحف: متحف الاثار بمكتبة الاسكندرية:

http://www.antiquities.gov.eg/DefaultAr/Museum/Pages/MuseumDetails.aspx?MusCode=17

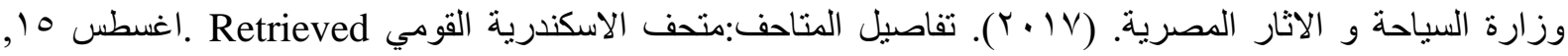
, from Y.r. .

http://www.antiquities.gov.eg/DefaultAr/Museum/Pages/MuseumDetails.aspx?MusCode 